\title{
Central Limit Theorems under additive deformations
}

Daniel J. Eck

Department of Statistics, University of Minnesota, 313 Ford Hall, 224 Church St SE, Minneapolis, MN 55455, USA.

Ian W. McKeague

Department of Biostatistics, Columbia University, 722 West 168th St. NY, NY 10032, USA.

\begin{abstract}
Additive deformations of statistical systems arise in various areas of physics. Classical central limit theory is then no longer applicable, even when standard independence assumptions are preserved. This paper investigates ways in which deformed algebraic operations lead to distinctive central limit theory. We establish some general central limit results that are applicable to a range of examples arising in nonextensive statistical mechanics, including the addition of momenta and velocities via Kaniadakis addition, and Tsallis addition. We also investigate extensions to random additive deformations, and find evidence (based on simulation studies) for a universal limit specific to each statistical system.
\end{abstract}

Keywords: Probability on Lie groups, Kaniadakis addition, Tsallis entropy

\section{Introduction}

The classical central limit theorem (CLT) is a cornerstones of statistics. In this article, we generalize this classical result to settings in which standard addition on the real line is replaced by a binary operation that satisfies Lie group properties. Additional mild smoothness assumptions are also imposed, allowing us to obtain explicit limiting distributions.

Our principal motivation comes from physics. As explained by [1], different Lie group operations on the real line are associated with distinctive forms of entropy that extend Boltzmann-Gibbs entropy, which corresponds to standard addition and classical central limit theory. Tsallis entropy applies to statistical systems exhibiting the features of long range dependence [2], and has been successfully applied, for example, in image thresholding [3], modeling debris flow [4], analyzing electromagnetic pre-seismic emissions [5], and modeling the distribution of momenta of cold atoms in optical lattices [6]. Kaniadakis entropy arises when combining momenta in special relativity [7, 8], and its associated central limit theory has recently been developed by [9], who showed that the limiting distributions take the form of hyperbolic functions of standard normals.

Email addresses: eckxx049@umn.edu (Daniel J. Eck), im2131@cumc.columbia.edu (Ian W. McKeague) 
There is a general formulation of the CLT on locally compact Lie groups due to [10], but conditions are placed on

\section{CLTs under additive deformations}

Our results extend the classical CLT on the real line to allow additive deformations of the following form. Standard addition is replaced by a group operation $\oplus$ defined on an open and possibly infinite interval $G$, with $(G, \oplus)$ assumed to be a Lie group under the usual topology on the real line. Since all Lie groups on the real line are isomorphic to their Lie algebra $(\mathbb{R},+)$, there exists an isomorphism $g: G \rightarrow \mathbb{R}$ (that is unique up to scalar multiples) such that

$$
g(x \oplus y)=g(x)+g(y)
$$

for all $x, y \in G$. In Lie group terminology, $g$ is called the "logarithmic" map, and its inverse $f=g^{-1}$ the "exponential" map. Let $e \in G$ be the identity, and denote $G_{e}=G-e$. We now give our main result showing that if $g$ has a second order Taylor expansion around $e$, in which the leading term is linear, then the CLT extends to $\oplus$-addition.

Theorem 1. Let $\left\{X_{i}\right\}$ be a sequence of iid $G_{e}$-valued mean-zero random variables with finite variance $\sigma^{2}$, and let $X_{n, i}=e+X_{i} / \sqrt{n}$. Suppose there exists a function $\rho: G_{e} \rightarrow \mathbb{R}^{+}$such that

$$
\begin{gathered}
\rho(x) \rightarrow 0 \text { as } x \rightarrow 0, \quad \rho(x / s) \leq M \text { for } x \in G_{e}, s \geq s_{0} \\
\left|g(e+x)-x-a x^{2}\right| \leq x^{2} \rho(x) \quad \text { for } x \in G_{e},
\end{gathered}
$$


where $a, s_{0}>1$ and $M>0$ are prespecified constants. Also suppose there exist constants $c_{1}, c_{2}, c_{3}$, and $s_{1}>0$, such that for all $x \in G_{e}$ and $s \geq s_{1}$,

$$
s|g(e+x / s)| \leq c_{1}|x| 1\left(|x| \geq c_{2}\right)+c_{3} .
$$

Then

$$
X_{n, 1} \oplus X_{n, 2} \oplus \ldots \oplus X_{n, n} \stackrel{\mathcal{D}}{\longrightarrow} f(Z)
$$

\section{Remarks:}

1. The key smoothness condition (3) in Theorem 1 is that $g$ has a parabolic local approximation at the identity $e$. The parabola can take the general form $x \mapsto a(x-e)^{2}+b(x-e)$, the only requirements being that it go through $(e, 0)$, since $g(e)=0$, and that $b \neq 0$ (so the leading term is linear). The coefficients $a$ and $b$, along with $\sigma^{2}$, determine the "bias" of the normal r.v. $Z$ that appears in the limit; for simplicity we stated the result just for the case $b=1$ (giving bias $a \sigma^{2}$ ), but the result extends to the general case, where the limit is $f\left(b Z_{b}\right)$ with $Z_{b} \sim N\left(a \sigma^{2} / b, \sigma^{2}\right)$. This follows from Theorem 1 with $a$ changed to $a / b$, and the maps $g$ and $f$ changed to $x \mapsto g(x) / b$ and $x \mapsto f(b x)$, respectively. When $g$ is locally approximated by a straight line $x \mapsto b(x-e)$ (i.e., $a=0$ ), there is no bias.

2. In Section 3 we will examine various examples in which we can find the logarithmic map $g$, along with its local parabolic approximation, leading to an explicit limit distribution. A classical and well-known instance arises in connection with the CLT for products of positive r.v.s, in which case $G=(0, \infty), x \oplus y=x y$ for $x, y \in G, e=1, g=\log , f=\exp$, and the limit distribution is log-normal. Specifically, our result gives $\prod_{i=1}^{n} X_{n, i} \stackrel{\mathcal{D}}{\longrightarrow} \exp (Z)$, where $Z \sim N\left(-\sigma^{2} / 2, \sigma^{2}\right)$, where $X_{i}>-1$ is assumed to have mean zero and finite variance $\sigma^{2}$. Condition (3) holds in this case by a Taylor series expansion of $x \mapsto \log (1+x)$ around $0 \in G_{e}=(-1, \infty)$, namely

$$
\left|\log (1+x)-x+x^{2} / 2\right| \leq x^{2} \rho(x), \quad x>-1,
$$

where $\rho(x)=|x /(1+x)|$ satisfies (2) with $M=1 /\left(s_{0}-1\right)$ for any $s_{0}>1$. This expansion is verified in Section 3.2.

3. Condition (4) was only used in the proof to allow dominated convergence arguments to be applied to $\sqrt{n} g\left(X_{1} / \sqrt{n}\right)$ and $n g\left(X_{1} / \sqrt{n}\right)^{2}$. However, if $X_{1}$ is assumed to have a finite fourth moment then (4) is not needed and the theorem continues to hold, as shown in Lemma 1 in the Supplementary Materials.

Proof of Theorem 1. For simplicity we assume that the identity $e=0$, so $G_{e}=G$, but the proof easily extends to the 
general case. By the definition of isomorphism, we have

$$
\begin{aligned}
X_{n, 1} \oplus X_{n, 2} \oplus \ldots \oplus X_{n, n} & =f\left[g\left(X_{n, 1}\right)+g\left(X_{n, 2}\right)+\ldots+g\left(X_{n, n}\right)\right] \\
& =f\left[\sum_{i=1}^{n}\left(g\left(X_{n, i}\right)-E g\left(X_{n, i}\right)\right)+n E g\left(X_{n, 1}\right)\right] .
\end{aligned}
$$

Therefore,

$$
X_{n, 1} \oplus X_{n, 2} \oplus \ldots \oplus X_{n, n}=f\left[T_{n}+n E g\left(X_{n, 1}\right)\right]
$$

where $T_{n}=\sum_{i=1}^{n}\left(g\left(X_{n, i}\right)-E g\left(X_{n, i}\right)\right)$. The Lindeberg-Feller theorem is used to find the asymptotic distribution of $T_{n}$. We first find the asymptotic variance of $T_{n}$. For $s>s_{0}$,

$$
\begin{aligned}
|s g(x / s)-x| & \leq\left|s g(x / s)-x-a x^{2} / s\right|+|a| x^{2} / s \\
& \leq \rho(x / s) x^{2} / s+|a| x^{2} / s \\
& \leq x^{2}(M+|a|) / s,
\end{aligned}
$$

where the last two inequalities follow from assumptions (2) and (3), so

$$
\lim _{s \rightarrow \infty} s g(x / s)=x
$$

for all $x \in G$. From assumption (4), we have $\left|\sqrt{n} g\left(X_{n, 1}\right)\right| \leq c_{1}\left|X_{1}\right| 1\left(\left|X_{1}\right| \geq c_{2}\right)+c_{3}$ and $\left[\sqrt{n} g\left(X_{n, 1}\right)\right]^{2} \leq 2 c_{1}^{2} X_{1}^{2}$ $+2 c_{3}^{2}$, so by dominated convergence and (8), $E\left[\sqrt{n} g\left(X_{n, 1}\right)\right] \rightarrow E X_{1}=0$ and $E\left[\sqrt{n} g\left(X_{n, 1}\right)\right]^{2} \rightarrow \sigma^{2}$, resulting in

$$
\operatorname{Var}\left(T_{n}\right)=n \operatorname{Var}\left(g\left(X_{n, 1}\right)\right)=E\left(\sqrt{n} g\left(X_{n, 1}\right)\right)^{2}-\left(E \sqrt{n} g\left(X_{n, 1}\right)\right)^{2} \rightarrow \sigma^{2} .
$$

We next check the Lindeberg condition. Fix $\varepsilon>0$ and note that

$$
\begin{aligned}
& \sum_{i=1}^{n} E\left[\left(g\left(X_{n, i}\right)-E g\left(X_{n, i}\right)\right)^{2} 1\left(\left|g\left(X_{n, i}\right)-E g\left(X_{n, i}\right)\right|>\varepsilon\right)\right] \\
& \leq \operatorname{Var}\left(T_{n}\right)-E\left[\left(\sqrt{n} g\left(X_{n, 1}\right)-E\left(\sqrt{n} g\left(X_{n, 1}\right)\right)\right)^{2} 1\left(\left(\sqrt{n} g\left(X_{n, 1}\right)-E\left(\sqrt{n} g\left(X_{n, 1}\right)\right)\right)^{2} \leq t\right)\right]
\end{aligned}
$$

provided $t \leq n \varepsilon^{2}$. The variance term above tends to $\sigma^{2}$ (as we have already seen). If $t$ is a fixed continuity point of the distribution of $X_{1}^{2}$ then the last term above tends to $-E X_{1}^{2} 1\left\{X_{1}^{2} \leq t\right\}$ by the continuous mapping theorem and dominated convergence, which in turn tends to $-\sigma^{2}$ as $t \rightarrow \infty$ by dominated convergence. Therefore the Lindeberg condition holds and $T_{n} \stackrel{\mathcal{D}}{\rightarrow} N\left(0, \sigma^{2}\right)$. The result then follows form (7) using Slutsky's lemma and the continuous mapping theorem provided we show $n E g\left(X_{n, 1}\right) \rightarrow a \sigma^{2}$. Using the zero mean property of $X_{1}$ and assumption (3) we have

$$
\begin{aligned}
\left|n E g\left(X_{n, 1}\right)-a \sigma^{2}\right| & \leq n E\left|g\left(X_{n, 1}\right)-X_{n, 1}-a X_{n, 1}^{2}\right| \\
& \leq n E\left[X_{n, 1}^{2} \rho\left(X_{n, 1}\right)\right]=E\left[X_{1}^{2} \rho\left(X_{1} / \sqrt{n}\right)\right]
\end{aligned}
$$


which tends to zero by (2) and dominated convergence. This completes the proof.

The assumption that $g$ has a parabolic approximation with a linear trend was crucial for Theorem 1 in the sense that it is the natural condition for the case of $\sqrt{n}$-normalization. We now show that higher-order approximations to $g$ also lead to CLTs, provided the normalization matches the order of the leading term in the approximation, and the sum is centered by a "drift" term. When the approximation to $g$ has no linear leading term, the deformation can cause the drift term to tend to infinity, so in general a tight limiting distribution is not possible without centering.

Theorem 2. Suppose there exists a function $\rho: G_{e} \rightarrow \mathbb{R}^{+}$satisfying (2) and

$$
\left|g(e+x)-\sum_{j=1}^{p} a_{j} x^{k_{j}}\right| \leq \rho(x) x^{k_{p}}
$$

for all $x \in G_{e}$, where the $a_{j}$ and $1 \leq k_{1}<k_{2}<\cdots<k_{p}$ are prespecified, with $a_{1}=1$. Assume

$$
\left|s g\left(e+s^{-1 / k_{1}} x\right)\right| \leq c_{1}|x|^{k_{1}} 1\left(|x| \geq c_{2}\right)+c_{3}
$$

for all $x \in G_{e}$ and $s>s_{1}$, where $c_{1}, c_{2}, c_{3}$ and $s_{1}>0$ are prespecified. Let $\left\{X_{i}\right\}$ be a sequence of iid $G_{e}-v a l u e d$ random variables with $E\left(X_{1}^{k_{1}}\right)=0$ and $\sigma^{2}=E\left(X_{1}^{2 k_{1}}\right)<\infty$. Let $X_{n, i}=e+n^{-1 /\left(2 k_{1}\right)} X_{i}$. Then

$$
X_{n, 1} \oplus X_{n, 2} \oplus \ldots \oplus X_{n, n} \ominus f\left(\mu_{n}\right) \stackrel{\mathcal{D}}{\longrightarrow} f(Z)
$$

where $Z \sim N\left(0, \sigma^{2}\right)$ and $\mu_{n}=n E g\left(X_{n, 1}\right)$.

The proof of Theorem 2 is included in the Supplementary Materials.

\section{Examples}

Statistical systems that can be described in terms of Boltzmann-Gibbs entropy are associated with classical addition. Since both the logarithmic and exponential maps are the identity in this setting, the conditions of Theorem 1 are satisfied trivially. It is immediately seen that our main theorem generalizes the classical CLT. In this section we present several examples that go beyond the classical setting.

\subsection{Kaniadakis addition}

Our result can be used to derive a relativistic CLT given by [9], who considered the case of Kaniadakis addition:

$$
x \stackrel{\kappa}{\oplus} y=x \sqrt{1+\kappa^{2} y^{2}}+y \sqrt{1+\kappa^{2} x^{2}},
$$

representing the addition of momenta in special relativity. The parameter $0<\kappa \leq 1$ is the reciprocal of the speed of light in the ambient space (when all variables are expressed in dimensionless units). The Lie group (R, $\stackrel{\kappa}{\oplus}$ ) has exponential map $f_{\kappa}(x)=\sinh (\kappa x) / \kappa$, and logarithmic map

$$
g_{\kappa}(x)=\frac{1}{\kappa} \sinh ^{-1}(\kappa x)=\frac{1}{\kappa} \log \left(\kappa x+\sqrt{1+\kappa^{2} x^{2}}\right) .
$$


Condition (3) can be checked using an inequality in [9], which yields

$$
\left|g_{\kappa}(x)-x\right| \leq x^{2} \rho(x)
$$

with $\rho(x)=\kappa \min (\kappa|x|, 1)$ for all $x \in \mathbb{R}$. This $\rho$ is bounded everywhere and $\rho(x) \rightarrow 0$ as $x \rightarrow 0$, so condition (2) holds. Further, $\left|g_{\kappa}(x)\right| \leq|x|$, so (4) holds. Therefore, all conditions of Theorem 1 are met, and we conclude that, for any iid sequence $\left\{X_{i}\right\}$ of mean-zero random variables with finite variance $\sigma^{2}$,

$$
X_{n, 1} \stackrel{\kappa}{\oplus} X_{n, 2} \stackrel{\kappa}{\oplus} \ldots \stackrel{\kappa}{\oplus} X_{n, n} \stackrel{\mathcal{D}}{\longrightarrow} \frac{1}{\kappa} \sinh (\kappa Z)
$$

65

where $Z \sim N\left(0, \sigma^{2}\right)$. In this setting, $Z$ has mean zero since 3 is satisfied with $a=0$. We have reached the same conclusion as [9, Theorem 1].

[9] also derived CLTs for velocity and energy using identities of [7] and the continuous mapping theorem to translate the corresponding CLT for momentum into velocity and energy. Our Theorem 1 provides a more direct approach. Velocities are combined according to the Einstein addition rule

$$
x \stackrel{v}{\oplus} y=\frac{x+y}{1+\kappa^{2} x y}
$$

for $x, y \in G=(-1 / \kappa, 1 / \kappa)$, and the corresponding Lie group $(G \stackrel{v}{\oplus})$ has exponential map $f_{v}(x)=\frac{1}{\kappa} \tanh (\kappa x)$ and logarithmic map

$$
g_{v}(x)=\frac{1}{\kappa} \tanh ^{-1}(\kappa x)=\frac{1}{2 \kappa} \log \left(\frac{1+\kappa x}{1-\kappa x}\right)
$$

We now verify that 3 holds:

$$
\left|g_{v}(x)-x\right| \leq \rho(x) x^{2}
$$

with $\rho(x)=|x /(1-\kappa|x|)|$. This $\rho$ obviously satisfies condition 22. The function $h(x)=x^{2} \rho(x)-\left|g_{v}(x)-x\right|$ has derivative

$$
h^{\prime}(x)=\left(\frac{x^{2}}{1-\kappa|x|}\left(\frac{\kappa x^{2}}{|x|(1-\kappa|x|)}+1\right)+\frac{\kappa^{2} x^{2}}{1-\kappa^{2} x^{2}}+\frac{2 x^{2}}{1-\kappa|x|}\right) \operatorname{sgn}(x),
$$

which is negative when $x<0$, positive when $x>0$. Since $h_{v}(0)=0$, it follows that $h(x) \geq 0$ for all $x \in G$, so condition 3 holds as claimed. Similarly, the derivative of $x \mapsto \kappa|x|-g_{v}(x)$, namely

$$
\kappa\left[1-\frac{|x|}{\kappa^{2} x^{2}-1}\right] \operatorname{sgn}(x),
$$

is negative for $x<0$ and positive for $x>0$, so condition (4) holds with $c_{1}=\kappa, c_{2}=c_{3}=0$. All the conditions of Theorem 1 are satisfied, and we conclude that if $\left\{X_{i}\right\}$ is an iid sequence of mean-zero $G$-valued random variables with variance $\sigma^{2}$ (which is necessarily finite), then

$$
X_{n, 1} \stackrel{v}{\oplus} X_{n, 2} \stackrel{v}{\oplus} \ldots \stackrel{v}{\oplus} X_{n, n} \stackrel{\mathcal{D}}{\longrightarrow} \frac{1}{\kappa} \tanh (\kappa Z)
$$

where $Z \sim N\left(0, \sigma^{2}\right)$. 


\subsection{Tsallis addition}

We next show that our theory leads to CLTs under addition associated with Tsallis entropy. Tsallis addition is a combination of standard addition and multiplication:

$$
x \stackrel{q}{\oplus} y=x+y+(1-q) x y,
$$

where $x, y \in G=(-1 /(1-q), \infty)$, and $0 \leq q<1$. The exponential map $f_{q}: \mathbb{R} \rightarrow G$ and respective logarithmic map $g_{q}$ are given by

$$
f_{q}(x)=\frac{\exp ((1-q) x)-1}{1-q}, \quad \text { and }, \quad g_{q}(x)=\frac{\log (1+(1-q) x)}{1-q},
$$

respectively. Condition $(3)$ of Theorem 1 holds by a Taylor series expansion of $g_{q}$ around $0 \in G$, namely

$$
\left|g_{q}(x)-x+(1-q) x^{2} / 2\right| \leq x^{2} \rho(x), \quad x>-1,
$$

where $\rho(x)=|x /(1+x)|$ and $a=-(1-q) / 2$. This expansion follows from 6 in Remark 1 , where we already noted that $\rho$ satisfies condition (2). Let

$$
h(x)=x^{2} \rho(x)-\left|\log (1+x)-x+x^{2} / 2\right| .
$$

The derivative

$$
h^{\prime}(x)=\left(\frac{x}{x+1}\right)^{3}\left|\frac{x+1}{x}\right|+2 x\left|\frac{x}{x+1}\right|-\frac{x^{2}}{x+1} \operatorname{sgn}\left(x^{2}-2 x+2 \log (1+x)\right)
$$

is negative for $-1<x<0$ and positive for $x>0$. Since $h(0)=0$, we then have $h(x) \geq 0$ for all $x \in G$, so (6) holds. Next we check that (4) holds for $g_{q}$ with $q=0$, from which the case of general $g_{q}$ follows immediately. First it is easy to check that $|s \log (1+x / s)| \leq|x|$ for all $s>s_{0}>1$ when $x>0$. Second, suppose $-1<x<0$. From the monotonicity of the $\operatorname{logarithm}, s \log (1-1 / s) \leq s \log (1+x / s) \leq 0$. Fix $\varepsilon>0$. As seen in the proof of Theorem 1 . condition (3) implies that $s g(x / s) \rightarrow x$ as $s \rightarrow \infty$. Thus, for some positive $s_{1}$, we have

$$
-1-\varepsilon \leq s \log (1-1 / s) \leq s \log (1+x / s) \leq \varepsilon
$$

for all $s>s_{1}$. Therefore condition (4) holds with

$$
s|\log (1+x / s)| \leq|x| 1(|x| \geq 1)+1+\varepsilon .
$$

All of the conditions of Theorem 1 are now verified for Tsallis addition, and we conclude that if $\left\{X_{i}\right\}$ is an iid $Z \sim N\left(-(1-q) \sigma^{2} / 2, \sigma^{2}\right)$.

Example 3.1. (Tsallis addition and the product of positive random variables.) The verification of the conditions of Theorem 1 for the product of iid positive random variables in Remark 1 overlaps with the verifications for Tsallis 
addition. This is because there is a direct connection between the two binary operations: $(1+x)(1+y)=1+x \stackrel{q}{\oplus} y$ for Tsallis addition with $q=0$. We can then derive the limit distribution of the product as a shifted version of the limit in the CLT under Tsallis addition. To see this, express $X_{n, i}$ in Remark 1 as $1+Y_{n, i}$, where $Y_{n, i}=X_{i} / \sqrt{n}$, so when $q=0$ we have $\prod_{i=1}^{n} X_{n, i}=1+Y_{n, 1} \stackrel{q}{\oplus} \cdots \stackrel{q}{\oplus} Y_{n, n}$.

It is now apparent that as a consequence of Theorem 1 distinct central limit theories are associated with BoltzmannGibbs, Kaniadakis, and Tsallis group entropies. Tsallis entropy is the only one of these to exhibit asymptotic bias in the sense that the normally distributed $Z$ appearing in the limit $f(Z)$ has non-zero mean. The bias is due to the presence of a second-order term in the Taylor expansion of the corresponding logarithmic map.

\subsection{Deformations via exponentiation}

We now provide an example that falls outside of the scope of Theorem 1, but that is covered by Theorem 2 which allows for more extreme types of deformations, provided the normalization of the summands is chosen appropriately. Consider the Lie group on $G=\mathbb{R}$ with binary operation

$$
x \stackrel{\alpha}{\oplus} y=\left(x^{\alpha}+y^{\alpha}\right)^{1 / \alpha},
$$

where $\alpha \geq 1$, the integer part of $\alpha$ is odd, and $x^{\alpha}=\exp [\alpha \log |x|] \operatorname{sgn}(x)$. The identity element is $e=0$, the exponential map $f_{\alpha}(x)=x^{1 / \alpha}$, and the logarithmic map $g_{\alpha}(x)=x^{\alpha}$. Condition 9 is satisfied with $\rho(x)=0$, ${ }_{85} p=1, a_{1}=1$ and $k_{1}=\alpha$. Condition 10 holds with $c_{1}=c_{2}=1$ and $c_{3}=0$. Let $\left\{X_{i}\right\}$ be an iid sequence of random variables with $E\left(X_{1}^{\alpha}\right)=0$ and $\sigma^{2}=E\left(X_{1}^{2 \alpha}\right)<\infty$ and put $X_{n, i}=n^{-1 /(2 \alpha)} X_{i}$. Then 111 holds, and since there is no drift in this case $\left(\mu_{n}=0\right)$, we conclude that $X_{n, 1} \stackrel{\alpha}{\oplus} X_{n, 2} \stackrel{\alpha}{\oplus} \ldots \stackrel{\alpha}{\oplus} X_{n, n} \stackrel{\mathcal{D}}{\longrightarrow} Z^{1 / \alpha}$ where $Z \sim N\left(0, \sigma^{2}\right)$.

\section{Random additive deformations}

A basic assumption of the central limit theory we have developed is that the additive deformation is fixed. In particular, under Tsallis and Kaniadakis addition, the deformations are determined by the parameters $q$ and $\kappa$. In this section, we present the results of a simulation study in which these parameters are allowed to be random. For applications of Kaniadakis addition, a random $\kappa$ can arise when there are local variations in the ambient space through which (relativistic) particles are moving. In typical applications of Tsallis addition, $q$ is regarded as a fitting parameter [3], so treating it as random provides a way of adjusting for uncertainty about its actual value.

Suppose the iid mean-zero random variables $X_{i}$, normalized as $X_{n, i}=X_{i} / \sqrt{n}$, are combined according to

$$
X_{n, 1} \stackrel{\kappa_{1}}{\oplus} X_{n, 2} \stackrel{\kappa_{2}}{\oplus} \cdots \stackrel{\kappa_{n-1}}{\oplus} X_{n, n}
$$

95

where the $\kappa_{i}$ are iid and $x \stackrel{\kappa}{\oplus} y=x+y+\kappa x y$, as in Tsallis addition with $\kappa=1-q$, or $x \stackrel{\kappa}{\oplus} y=x \sqrt{1+\kappa^{2} y^{2}}+$ $y \sqrt{1+\kappa^{2} x^{2}}$ as in Kaniadakis addition. Associativity no longer holds, so we need to specify the order of operations in 14. The order is assumed to be from left to right, as in $\left(\left(\ldots\left(\left(X_{n, 1} \stackrel{\kappa_{1}}{\oplus} X_{n, 2}\right) \stackrel{\kappa_{2}}{\oplus} X_{n, 3}\right) \stackrel{\kappa_{3}}{\oplus} \ldots\right) \stackrel{\kappa_{n-1}}{\oplus} X_{n, n}\right.$. We 
have found from simulations that the sampling distribution appears to be the same in the reverse order, or in fact in any order, but we do not have a proof of this.

It would be interesting to establish the existence of limiting distributions of sums of the form [14], that are universal in the sense that they do not depend on the distributions of $X_{i}$ or $\kappa_{i}$, but only on certain features of these distributions (such as their mean an variance) and on features of the deformation. This appears to be a very challenging problem. Our simulation studies, however, do shed some light on the question of whether such a universal limit exists.

A broad simulation study provides support for this finding across a wide range of scenarios. The behavior of sampling distributions for (14) is investigated for two data generating models with six additive parameter distributions each for both Tsallis and Kaniadakis addition. The sampling distributions corresponding to each scenario are constructed with one million samples of size $n=2000$. The settings and empirical results are displayed in Table 1 in the Supplementary Materials. It should be noted that the distribution of the $X_{i}$ has been standardized and the additive parameter distributions have been shifted and scaled so that the mean is $1 / 2$ and the standard deviation is 0.10 . The densities of the sampled random sums from Table 1 are plotted in Figure 1. The curves in both panels of Figure 1 provide strong visual evidence for the existence of a universal limit law.

The numerical summaries in Table 1 further support the visual evidence in favor of the existence of a universal limit law. A Kolmogorov-Smirnov test between each sampling distribution in Table 1 and a sampling distribution with fixed $\kappa=q=1 / 2$ shows no significant difference at any reasonable testing level. Shapiro-Wilks tests between each sampling distribution in Table 1 and the asymptotic log-normal (Tsallis case) and sinh-normal (Kanidakis case) distributions also provide evidence in favor of a universal limit law. The shapiro. test function used to implement the Shapiro-Wilks tests in R can only handle a maximum of five thousand entries. As a result of this, we repeatedly sample five thousand entries from the million possible values a total of ten thousand times and report the proportion of p-values exceeding 0.05 . What is striking about this procedure is the vast differences in the proportion of p-values exceeding 0.05 across addition operations and data generating distributions alike. The exponential data generating model and Tsallis addition are seen to be further away from asymptopia than the uniform data generating model and Kaniadakis addition respectively.

\section{Discussion}

Our theorems extend classical central limit theory to cases in which random variables are combined with a binary operation satisfying Lie group properties. We have shown that different algebraic deformations with distinct isomorphisms yield different limiting distributions. The three statistical systems considered, the combination of velocity mentioned in [9], and $\alpha$-norms of random variables all arise as special cases of our extended central limit theorems.

Theorem 1 in [9] provided the inspiration for the development of our theory. However, [9, Theorem 1] arises as a very special case of our theorems. In particular, $g$ has a local linear approximation instead of a local parabolic approximation, $g: \mathbb{R} \rightarrow \mathbb{R}$, and $\rho(x)$ is bounded everywhere. However, these properties do not hold in generality 

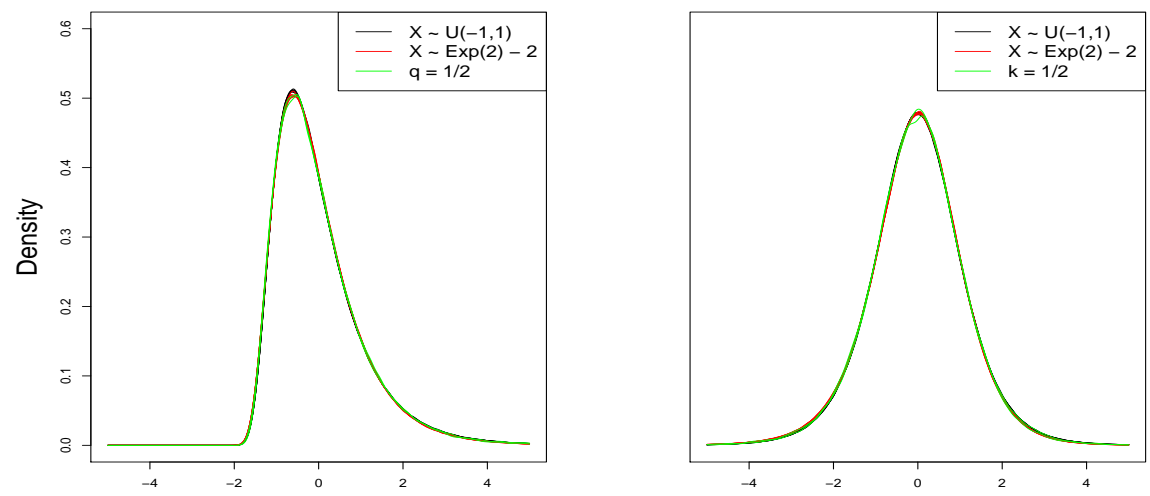

Figure 1: Evidence for the existence of a universal limit law. The sampling distributions for the simulation settings in Table 1 are plotted here. The left panel displays the density curves corresponding to the Tsallis case. The right panel displays the density curves corresponding to the Kaniadakis case. The green lines correspond to density curves for the fixed $q=\kappa=1 / 2$ case for both data generating distributions and both addition operations.

and they are relaxed in the present work. As a consequence, limiting normal distributions in $[5]$ are not necessarily mean-zero and operations not defined over all of $\mathbb{R}$ have extensions. Theorem 2 then relaxes the requirement that $g$ has a local parabolic approximation. In this Theorem, the approximation to $g$ is then more general than a second order Taylor expansion.

The generality of our theory allows us to find the limiting distribution when random elements are combined via Tsallis addition. We are not the only authors to investigate limiting distributions in this setting. [11] presents a $q$-central limit theory motivated by nonextensive statistical mechanics and Tsallis addition. In their setting, random variables are correlated and generalizations of independence ( $q_{k}$-independence) are characterized by the Fourier transform defined within [11, section 2.4 and definition 3.2]. They show that a $q_{k}$-independent sequence $q_{k}$ converges to a $q_{k-1}$-normal distribution[11, Theorem 1]. The statistical context of this result is not immediately apparent. Our theorems show that classical central limit theory can be extended to nonextensive statistical mechanics by exchanging standard addition with Tsallis addition when random variables meet classical assumptions.

The simulation studies within provide strong evidence in favor of the existence of a universal limit law. This universal limit law states that when additive parameters are themselves random, the sampling distribution of (14) will converge to that of (5) where the fitting parameter in (5) is the mean of the random additive parameters in (14). The evidence suggests that random deformations converge to the asymptotic distributions with fixed parameters given by our theorems. In addition, the output in Table 1 suggests that some data generating mechanisms and addition operations approach the universal limit law faster than others.

\section{Acknowledgments}




\section{References}

[1] P. Tempesta, Group entropies, correlation laws and zeta functions, Phys. Rev. E 84 (2011) 021121.

[2] C. Tsallis, Possible generalization of Boltzmann-Gibbs statistics, J. Statist. Phys. 52 (1988) 479-487.

[3] M. Portes de Albuquerque, I. A. Esquef, A. R. Gesualdi Mello, M. Portes de Albuquerque, Image thresholding using Tsallis entropy, Pat. Recog. Let. 25 (2004) 1059-1065.

[4] V. P. Singh, H. Cui, Modeling sediment concentration in debris flow by Tsallis entropy, Phys. A: Statist. Mech. Appl. 420 (2015) 49-58.

[5] S. M. Potirakis, G. Minadakis, K. Eftaxias, Analysis of electromagnetic pre-seismic emissions using Fisher information and Tsallis entropy, Phys. A: Statist. Mech. Appl. 391 (2012) 300-306.

[6] P. Douglas, S. Bergamini, F. Renzoni, Tunable Tsallis distributions in dissipative optical lattices, Phys. Rev. Let. 96 (2006) 110601.

[7] G. Kaniadakis, Towards a relativistic statistical theory, Phys. A: Statist. Mech. Appl. 365 (2006) 17-23.

[8] G. Kaniadakis, Theoretical foundations and mathematical formalism of the power-law tailed statistical distributions, Entropy 15 (2013) 3983-4010.

[9] I. W. McKeague, Central limit theorems under special relativity, Statist. Prob. Let. 99 (2015) 149-155.

[10] D. Wehn, Probabilities on Lie groups, Proc. Nat. Acad. Sci. U.S.A. 48 (1962) 791-795.

[11] S. Umarov, C. Tsallis, S. Steinberg, On a $q$-central limit theorem consistent with nonextensive statistical mechanics, Mil. J. Math. 76 (2008) 307-328. 